\title{
Light-induced biological waveguides
}

\author{
Anna Bezryadina*a, Rekha Gautam ${ }^{\text {b, }}$, Nicolas Perez ${ }^{\mathrm{a}}$, Tobias Hansson ${ }^{\mathrm{d}}$, Yinxiao Xiang ${ }^{\mathrm{b}, \mathrm{e}}$, Josh \\ Lamstein $^{\mathrm{b}}$, Benjamin Wetzel ${ }^{\mathrm{d}, \mathrm{f}}$, Roberto Morandotti ${ }^{\mathrm{d}}$, Zhigang Chen ${ }^{\mathrm{b}, \mathrm{e}}$ \\ aDepartment of Physics and Astronomy, California State University Northridge, Northridge, \\ California, 91330, USA \\ ${ }^{b}$ Department of Physics and Astronomy, San Francisco State University, San Francisco, California \\ 94132, USA \\ ${ }^{c}$ Department of Biomedical Engineering, Vanderbilt University, Nashville, Tennessee, 37240, USA \\ ${ }^{\mathrm{d} I n s t i t u t ~ N a t i o n a l ~ d e ~ l a ~ R e c h e r c h e ~ S c i e n t i f i q u e, ~ U n i v e r s i t e ́ ~ d u ~ Q u e ́ b e c, ~ V a r e n n e s, ~ Q u e ́ b e c ~ J 3 X ~ 1 S 2, ~}$ \\ Canada
}

${ }^{\mathrm{e}}$ MOE Key Lab of Weak-Light Nonlinear Photonics, TEDA Applied Physics Institute and School of Physics, Nankai University, Tianjin 300457, China

fSchool of Mathematical and Physical Sciences, University of Sussex, 10 Sussex House, Falmer, Brighton BN1 9RH, United Kingdom

\begin{abstract}
Using light, living cells can be manipulated to form several centimeter long waveguide structures, capable of guiding light through scattering media. Here, we will discuss some results of self-trapping and guiding of light in biological suspensions of different cells, including cyanobacteria, E. coli, and red blood cells. A forward-scattering theoretical model is developed which helps understand the experimental observations. Formed waveguides can provide effective guidance for weaker light through scattered bio-soft-matter. The ability to transmit light through turbid fluids with low loss could open up the possibilities for deep-tissue imaging, as well as noninvasive treatment and diagnostics.
\end{abstract}

Keywords: biological waveguide, biological fiber, optical trapping, nonlinear optics effect, self-guiding, cyanobacteria, red blood cells, soliton.

\section{Introduction}

Understanding light-matter interaction in biological media has been focus of widespread studies since Ashkin's early work of optically trapping [1-2]. The study of nonlinear processes in biological samples is important for developing new methods for biophotonics, optofludics, imaging, and life-science. In the last decade, significant work was done to study deep-penetration of light and formation of optical spatial solitons in dielectric and metallic colloidal suspensions with tunable polarizability [3-6]. Similarly, with nonlinear optics, it is possible for light to self-induce a waveguide in biological media.

\section{Results and Discussion}

In this work, we discuss formation of waveguide structures in biological suspensions for a range of wavelengths. Specifically, several centimeters long biological waveguides, with no significant photodamage of the cells, have been successfully demonstrated in colloidal suspensions of cyanobacteria, E. coli, and red blood cells (RBCs) [7-9]. The details of the experimental setup and sample preparation have been published in the previous work [4,7]. Typical experimental results are presented in Figure 1, where cyanobacteria in seawater form a waveguide of light due to nonlinear self-trapping and self-focusing of light [7]. 

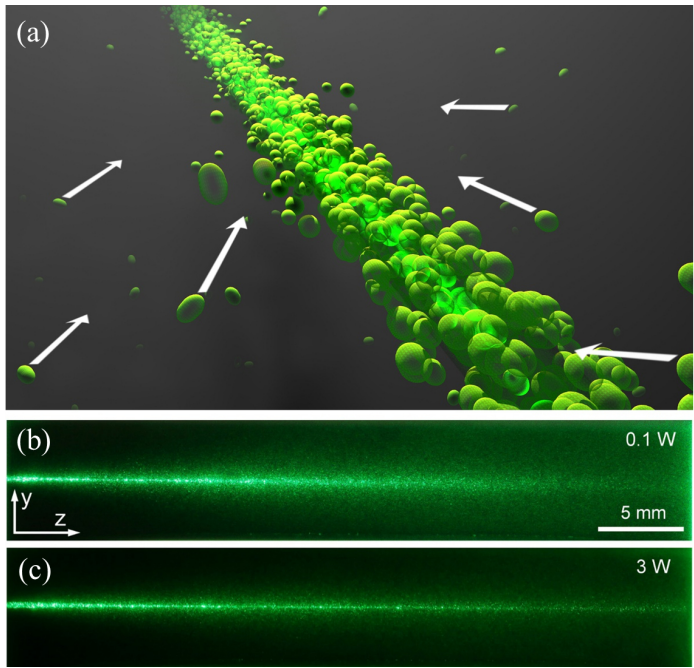

Figure 1. (a) Illustration of bacteria trapping and creating waveguide of light. (b,c) Side view of laser propagation in bacteria suspension at low and high laser power. The beam undergoes linear diffraction and scattering at low and experiences nonlinear selftrapping at high power [7].

According to the forward-scattering theoretical model, cells in colloidal suspensions get attracted toward the center of the beam due to the optical gradient force, and simultaneously move along the laser beam due to the scattering force [7]. Since living cells usually have a slightly higher index of refraction than the surrounding media, the optical force-induced nonlinearity leads to a self-lensing effect along the beam path, which allows for the formation of a biological "fiber".

Often, in biomedical and opto-fluidic micromanipulation studies the specific absorption bands need to be either targeted or avoided. However, sometimes the preferred wavelengths may not be suitable to exhibit nonlinear self-guiding. To overcome this challenge, a master/slave-type nonlinear coupling is implemented, where formed biological waveguides provide effective guidance for a weaker laser beam [6]. A strong optical beam (a pump beam) at $532 \mathrm{~nm}$ wavelength creates a selfinduced waveguide that guides a weak NIR optical beam (a probe beam) at various wavelengths. Figure 2 illustrates guiding a low power probe beam ("slave" beam) at different wavelengths by a green pump beam (master beam) in sheep RBC suspensions [9].

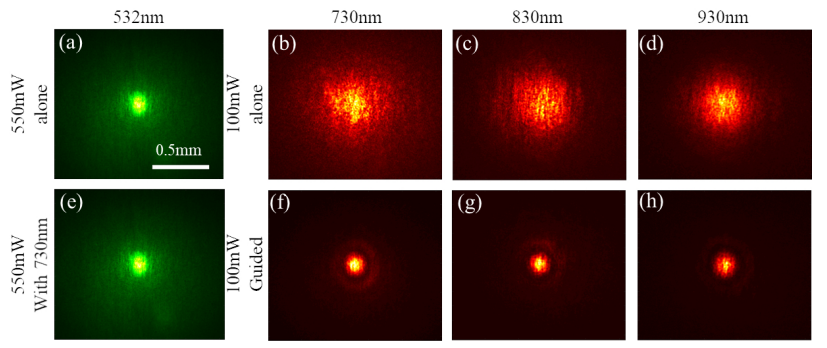

Figure 2. Master/slave guiding in RBC suspensions.[9] (a, e) Self-trapped green pump beam alone and with the probe beam at $550 \mathrm{~mW}$ laser power. (b-d) Probe beams $(730 \mathrm{~nm}, 830 \mathrm{~nm}, 930 \mathrm{~nm})$ alone in the linear diffracted regime at $100 \mathrm{~mW}$ laser power. (f-h)

Guiding of the probe beams by the green pump beam.

\section{Conclusion}

Waveguides in different biological suspensions over a few centimeters have been demonstrated. Furthermore, formed waveguides can guide light that has low nonlinear response itself in biological suspension. The ability to transmit light through turbid fluids at a wide range of wavelengths with low loss brings about new approaches for deep-tissue imaging, diagnostics, and treatments.

\section{References}

[1] Ashkin, A., "Acceleration and Trapping of particles by radiation pressure," Phys. Rev.Lett., 24, 156 (1970).

[2] Ashkin, A. and Dziedzic, J., "Optical trapping and manipulation of viruses and bacteria,” Science 235, 1517 (1987).

[3]El-Ganainy, R., Christodoulides, D., Rotschild, C. and Segev, M., "Soliton dynamics and self-induced transparency in nonlinear nanosuspensions," Opt. Express 15, 10207-10218 (2007).

[4]Man, M. et al., "Optical nonlinearities and enhanced light transmission in soft-matter systems with tunable polarizabilities," Phys. Rev. Lett. 111, 218302 (2013).

[5]Fardad, S., Salandrino, A., Heinrich, M., Zhang, P., Chen, Z. and Christodoulides, D.N., "Plasmonic resonant solitons in metallic nanosuspensions," Nano Lett. 14, 2498-2504 (2014).

[6]Kelly, T.S., Ren, Y.-X., Samadi, A., Bezryadina, A., Christodoulides, D. and Chen, Z., "Guiding and nonlinear coupling of light in plasmonic nanosuspensions," Opt. Lett. 41, 3817 (2016).

[7]Bezryadina, A. et al., "Nonlinear Self-Action of Light through Biological Suspensions,” Phys. Rev. Letts 119, 058101 (2017). 
[8] Gautam, R. et al. "Tunable optical nonlinearity and self-guiding of light through human red blood cells," Light: Sci. Appl. 8(1), 31 (2019).

[9]Perez, N., Chambers, J., Chen, Z. and Bezryadina, A., "Nonlinear self-trapping and guiding of light at different wavelengths with sheep blood," in submission (2020). 